\title{
Test Vehicle Speed Error as a Function of Tire Pressure
}

\author{
Andriy Dyukov \\ School of Mathematical and Geospatial Sciences, RMIT University, Melbourne, Victoria 3806, Australia
}

\begin{abstract}
The main objective of this study is to determine how speed errors of test vehicles depend on tire pressure. In order to achieve this, experiments were conducted to find out how tire pressure depends on driving speed and how distance measurements depend on tire pressure. It is demonstrated that hot tires might have their pressure increased by $6 \%$ at $110 \mathrm{~km} / \mathrm{h}$ speed relative to nominal tire pressure. Subsequently, experiments conducted on a surveyed test track proved that hot tires might increase speed measurement errors by $0.03 \%$, whereas under-inflated tires might cause several times higher speed errors. It is critical that test vehicles involved in speed measurements have nominal tire pressure when tires are cold. In such instances, the uncertainty of speed measurements component related to tire pressure is relatively low. This research allowed quantifying the tire pressure error component for a specific test vehicle, while the experimental approach might be used for any other vehicles.
\end{abstract}

Key words: Speed, measurement, uncertainty, tire, pressure.

\section{Introduction}

Test vehicles are used in a number of industries to measure speed. For example, speed cameras might be tested by test vehicles with the subsequent comparison of camera measurements against speed values produced by test vehicles. Also, GPS (Global Positioning System) receivers might be tested for their speed accuracy when the same comparison between the reference speed and vehicle speed records would apply. However, in all such test cases, test vehicles shall be accurate enough, which emphasize an importance of understanding their UOM (Uncertainty Of Measurements) and minimizing the UOM components.

One of the components influencing the UOM is the tire pressure. It is a common knowledge that changes in tire pressure may influence the correctness of speed measurements. At the same time little practical research results are published revealing how speed accuracy measurements depend on tire pressure. For example, in Ref. [1], it is mentioned that with tire pressure dropped, the measured speed would also drop. However, the magnitude of speed error is unknown.

Corresponding author: Andriy Dyukov, M.Sc. student, research fields: electronics, speed measurement and GNSS.
Also, it is unclear what would happen if the tire pressure increases. On the other hand, it is also known that the higher the speed, the hotter the tires. If a tire becomes hot, its pressure would increase and, as a consequence, the speed measurement conducted by the test vehicle becomes more inaccurate.

Therefore, it is important to investigate the dependency between the tire pressure and speed accuracy and quantify potential errors of test vehicles depending on their speed and changes in tire pressure at higher speeds.

\section{Method}

\subsection{General}

To determine the dependency of speed error on tire pressure, the following steps would be undertaken:

(1) determination of possible changes in tire pressure while a vehicle is driven with different speeds;

(2) determination of changes in distance measurement by the speed measurement system of the test vehicle depending on tire pressure;

(3) estimation of speed errors depending on tire pressure. 


\subsection{Speed Measurement Systems Based on Shaft Encoder and Their UOM}

Speed measurement systems based on shaft encoder installed on a wheel of test vehicles are widely used in the industry. For example, in Ref. [2], a speed measurement system is described based on Dynapar encoder producing 200 pulses per revolution of a wheel. In general, the structure of the speed measurement system based on shaft encoder looks as shown in Fig. 1.

In this system, the speed calculation unit counts pulses from the speed/distance sensor which represents a shaft encoder installed on a wheel, within a specific time. This time might be, for example, $1 \mathrm{~s}$ and is derived from a timing synchronization device (Fig. 1). Therefore, every second the speed calculation unit calculates speed based on the number of pulses from the shaft encoder as per the following equation:

$$
V=N \times K
$$

where:

$V$ is the calculated speed in $\mathrm{km} / \mathrm{h}$;

$N$ is the number of pulses counted within $1 \mathrm{~s}$;

$K$ is the calibration factor which depends on circumference of a wheel and the number of pulses of shaft encoder produced for one revolution of a wheel.

To minimize speed errors, some measures might be implemented, such as: deriving $1 \mathrm{~s}$ heartbeats from UTC (universal coordinated time) calculated by GPS receivers (Fig. 2), increasing the number of pulses produced by shaft encoder per revolution, etc. After implementation of such measures, the diagram of the speed measurement system might look as follows:

In this case, the UOM of speed measurements would have the following components in place: resolution of the speed measurement system, calibration uncertainty, timing interval uncertainty, tire pressure uncertainty due to changes in tire pressure while driving and some other components. The UOM of the test vehicle might be assessed in general by using proper techniques, for example, as

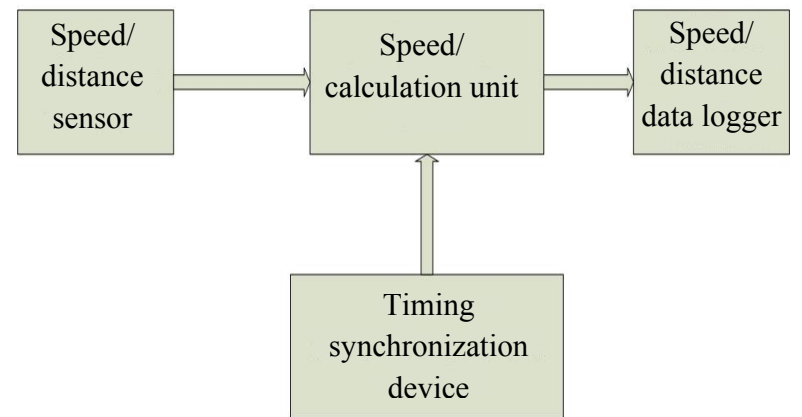

Fig. 1 Speed measurement system based on shaft encoder.

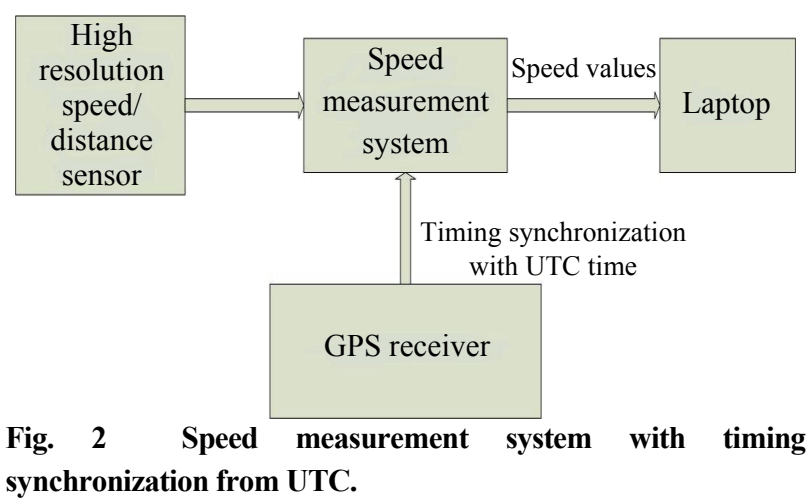

described in Ref. [3]. The task of this research is to investigate the UOM component related to tire pressure changes while driving. Quantification of this component would allow calculating the combined UOM of the test vehicle and assessing the magnitude of this particular component.

It is thought that in one rotation of a tire, the car moves forward by a distance equal to a circumference of a tire. In the case of a deflated tire, the rolling circumference might be reduced so much that it would be hard to guarantee that the distance the car moves forward with one rotation of the axle would be close to the distance that should be covered based on the circumference of the tire. However, if the tire pressure is initially installed to a nominal, it would go up rather than down while driving. In this instance, it is unclear how the rolling circumference grows. Testing of distance covered by a wheel with different tire pressures may reveal the result. To be accurate, this test needs to be conducted over a long distance, but that would also cause more constraints on measurement, such as: the distance is required to be properly surveyed and the speed measurement system 
should be able to operate in distance measurement mode only. To address the first issue, a distance of $361.3 \mathrm{~m}$ was precisely surveyed with the accuracy better than $1 \mathrm{~mm}$. To address the second issue, the speed measurement system of the test vehicle was designed in such a way that it could measure distance while operating in a distance measurement mode as shown in Fig. 3.

In this instance, as soon as driving starts, the system counts pulses coming from a shaft encoder installed on a wheel and using calibration factor $K$ recalculates the number of pulses into distance, incrementally logging distance values to a laptop. This principle would further be used to test a dependency between the tire pressure and distance measured.

\section{Results}

\subsection{The Link between the Tire Pressure and Speed}

The first step in investigation of how accurate the test vehicle might be depending on tire pressure is to reveal how hot tires might change the tire pressure.

To uncover the above dependency, an experiment was conducted where a test vehicle was driven through a number of speeds with tire pressure gauges installed on each wheel with radio transmitters embedded in each gauge. Fig. 4 below shows the principle of operation of the system during the experiment.

The system consists of four sensors installed on wheels while such sensors are capable to measure tire pressure on the move and transmit the results through radio frequency to the instrument panel. The latter can display pressures in sequence for each wheel or log pressures to a laptop via RS232 port embedded to the instrument panel. A number of such systems are produced by PressurePro in the US [4] and the 10 wheel monitor part \#ALTGPM10 was used for such experiments.

A vehicle Mazda3 was driven from $0 \mathrm{~km} / \mathrm{h}$ up to $110 \mathrm{~km} / \mathrm{h}$ with an initial tire pressure equal to 32 psi before driving, while 32 psi represents a

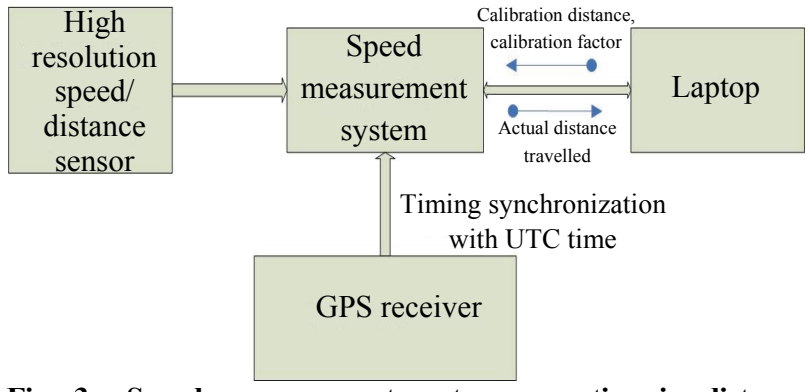

Fig. 3 Speed measurement system operating in distance measurement mode.

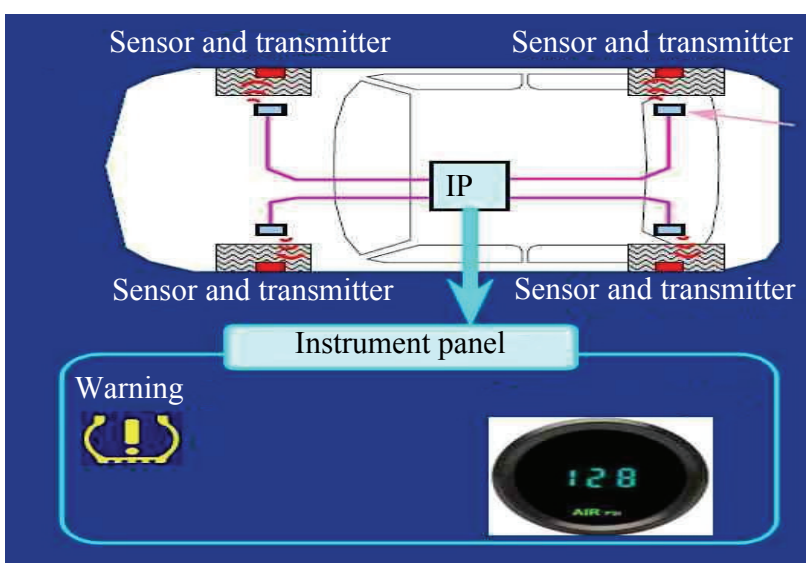

Fig. 4 Tire pressure measurement on the move.

nominal pressure for Mazda3. Further analysis of the log-file of tire pressure depending on speed revealed that at higher speeds tires are getting hotter which translates in changes of pressure from $32 \mathrm{psi}$ at $0 \mathrm{~km} / \mathrm{h}$ up to $34 \mathrm{psi}$ at $110 \mathrm{~km} / \mathrm{h}$. This essentially means that pressure increased by about $6 \%$ from its original nominal value. Quantifying the actual changes in tire pressure depending on how fast the test vehicle moved allowed to progress to the next stage and estimate potential error in speed measurements depending on tire pressure.

\subsection{Distance Measurement Depending on Tire}

\section{Pressure}

In the majority of test vehicles, speed is calculated as distance over time. Therefore, to understand how hot tires contribute to distance measurement errors, an experiment was conducted with a number of drives through a surveyed distance with different tire pressures: nominal and nominal $\pm 10 \%$. Such variations would cover a possible tire pressure increase while 
driving at high speed as shown in Section 3.1.

The site for experiments represented a straight section of the side road with the surveyed part of $361.3 \mathrm{~m}$ shown in Fig. 5. This site was surveyed with the use of electronic distance measurement methodology to the accuracy better than $1 \mathrm{~mm}$, which was achieved by the use of optical survey equipment Leica FlexLine [5]. This section of the road is actually going in parallel to Princess Hwy in Noble Park, Vic, Australia, but with almost no traffic. During subsequent experiments, the test vehicle was driven from $\mathrm{A}$ to $\mathrm{B}$ covering $361.3 \mathrm{~m}$ with the speed measurement system working in distance measurement mode as described in Section 2.2.

In this investigation, a vehicle Mazda3 was used which had 195/56 R15 tires, whereas a shaft encoder represented WDG $58 \mathrm{H}$ encoder manufactured by Wachendorff Automation Gmbh \& Co producing 2,048 pulses per revolution. This would equal to 948.9 pulses per meter if the tire pressure is nominal and the circumference of a tire equals to ideal. For such tires, the circumference ideally equals to 1,993.34 $\mathrm{mm}$. The speed measurement system was an in-house design and build.

Considering that the distance covered by a test vehicle in the experiment is $361.3 \mathrm{~m}$ and the number of pulses produced by the encoder per meter is 948.9, for tires with nominal tire pressure, ideally, the number of pulses counted for the test run would be as follows:

$$
N o=D \times K
$$

where:

No is the number of pulses counted within the test run;

$D$ is the test distance;

$K$ is the calibration factor.

Initially at Point A (Fig. 5), an alignment of the front tire to the start point is made as shown in Fig. 6. Then, at the start of test drive, a tester issues a command to the speed measurement system advising it to work in distance measurement mode with the respective calibration factor which is counted for ideal tires with 32 psi pressure.

As soon as vehicle moves, The system starts counting pulses from the high resolution shaft encoder and logging the current distance as soon as a test vehicle drives from A to B. As soon as Point B is reached, the test vehicle is stopped, a tester issues a command "finish" to the speed measurement system operating in distance mode and the distance covered with the certain tire pressure recorded.

The following results were obtained for 361.3-m driving distance for different tire pressures:

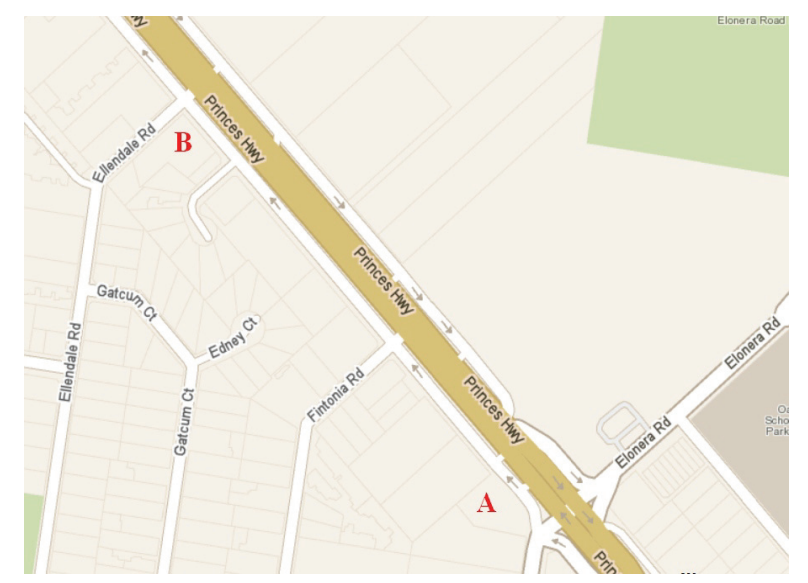

Fig. 5 Test site.

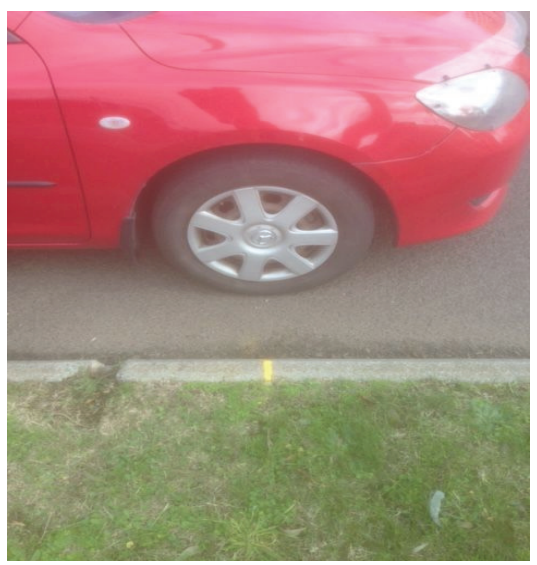

Fig. 6 Aligning a wheel at the start of the test.

Table 1 Test results for different tire pressures

\begin{tabular}{lll}
\hline Tire pressure (psi) & $\begin{array}{l}\text { Average distance } \\
\text { measured }(\mathrm{m})\end{array}$ & $\begin{array}{l}\text { Number of test } \\
\text { drives }\end{array}$ \\
\hline 28 & 362.0 & 10 \\
32 (nominal) & 361.3 & 10 \\
35 & 361.2 & 10 \\
\hline
\end{tabular}




\section{Discussion}

It appears that inner plies of a tire, particularly for a belted tire, would not stretch or compress a lot under circumstances. This is because the steel belt of a tire remains the same regardless of air pressure. When a tire is under-inflated, a tire thread is deformed to be flat against the ground and the tire flat spot, or footprint, increases with reduction of air pressure. Therefore, as the tire thread is deformed to be flat against the ground, the outermost part of it is slightly compressed to be shortened to the same length as the steel belt over the length of the flat area. This then accounts for the slightly reduced travel distance in relation to the tire circumference. It is visible that under-inflation causes a bigger error than over-inflation. Therefore, maintaining a proper initial tire pressure equal to nominal is important. If this condition is met, the subsequent speed error caused by changes in pressure by $10 \%$ might represent $0.03 \%$ of the actual speed, considering the ratio of 361.3 to 361.2. For example, at $100 \mathrm{~km} / \mathrm{h}$, an increase by $10 \%$ in tire pressure may cause a speed error up to just 0.03 $\mathrm{km} / \mathrm{h}$.

\section{Conclusions}

This research proves that under-inflation is much more problematic than over-inflation as far as speed measurements are concerned. More importantly, if the initial tire pressure is adjusted to a nominal, increases in tire pressure while driving might be up to $6 \%$ for a specific type of vehicle. Such increases would translate to a test vehicle inaccuracy of no more than $0.03 \%$ of the actual speed. The above value allows quantifying the UOM component related to speed errors depending on tire pressure for test vehicles participating in high accuracy speed measurements.

\section{References}

[1] Hardy, S. P. 2015. "Speedometer Inaccuracy, Radar Tolerances and the Law." Personal homepage of Hardy, S. P. Accessed October 13, 2015. http://www.trafficlaw.com.au/speedos.html.

[2] Wishwanathan, R. 2005. "Evaluation of Ground Speed Sensing Devices under Varying Ground Surface Conditions." M.Sc. thesis, Oklahoma State University.

[3] NASA (National Aeronautics and Space Administration). 2010. Estimation and Evaluation of Measurement Decision Risk, NASA Measurement Quality Assurance Handbook. Washington, D.C.: NASA.

[4] Advantage Enterprises, Inc. 2012. "PressurePro Product Catalog." Advantage Enterprises, Inc. Accessed October 15, 2015. http://advantagepressurepro.com/images/upload /FullCatalogJune12.pdf.

[5] Leica Geosystems AG. Leica FlexLine TS02/TS06/TS09 User Manual. Heerbrugg: Leica Geosystems AG. 